\title{
Postural stability is altered by the stimulation of pain but not warm receptors in humans Jean-Sébastien Blouin ${ }^{1}$, Philippe Corbeil ${ }^{1,2}$ and Normand Teasdale*1
}

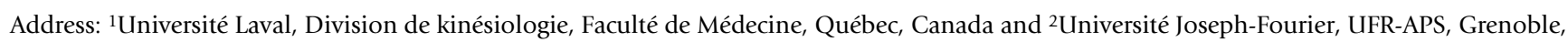
France

Email: Jean-Sébastien Blouin - jsblouin@kin.msp.ulaval.ca; Philippe Corbeil - philippe.corbeil@kin.msp.ulaval.ca; Normand Teasdale* - normand.teasdale@kin.msp.ulaval.ca

* Corresponding author

Published: 17 October 2003

BMC Musculoskeletal Disorders 2003, 4:23
Received: II August 2003

Accepted: 17 October 2003

This article is available from: http://www.biomedcentral.com/I47/-2474/4/23

(C) 2003 Blouin et al; licensee BioMed Central Ltd. This is an Open Access article: verbatim copying and redistribution of this article are permitted in all media for any purpose, provided this notice is preserved along with the article's original URL.

\begin{abstract}
Background: It is now recognized that large diameter myelinated afferents provide the primary source of lower limb proprioceptive information for maintaining an upright standing position. Small diameter afferents transmitting noxious stimuli, however, can also influence motor behaviors. Despite the possible influence of pain on motor behaviors, the effects of pain on the postural control system have not been well documented.

Methods: Two cutaneous heat stimulations (experiment I: non-noxious 40 degrees C; experiment 2: noxious 45 degrees $C$ ) were applied bilaterally on the calves of the subject with two thermal grills to stimulate $A$ delta and $C$ warm receptors and nociceptors in order to examine their effects on postural stability. The non-noxious stimulation induced a gentle sensation of warmth and the noxious stimulation induced a perception of heat pain (visual analogue scores of 0 and $46 \mathrm{~mm}$, respectively). For both experiments, ten healthy young adults were tested with and without heat stimulations of the lower limbs while standing upright on a force platform with eyes open, eyes closed and eyes closed with tendon co-vibration of tibialis anterior and triceps surae muscles. The center of pressure displacements were analyzed to examine how both stimulations affected the regulation of quiet standing and if the effects were exacerbated when vision was removed or ankle proprioception perturbed.

Results: The stimulation of the warm receptors ( 40 degrees $C$ ) did not induce any postural deterioration. With pain (45 degrees $C$ ), subjects showed a significant increase in standard deviation, range and mean velocity of postural oscillations as well as standard deviation of the center of pressure velocity. The effects of heat pain were exacerbated when subjects had both their eyes closed and ankle tendons vibrated (increased standard deviation of the center of pressure velocity and mean velocity of the center of pressure).

Conclusions: A non-noxious stimulation (40 degrees $\mathrm{C}$ ) of the small diameter afferents is not a sufficiently intense sensory stimulation to alter the control of posture. A painful stimulation (45 degrees C) of the skin thermoreceptors, however, yielded a deterioration of the postural control system. The observed deteriorating effects of the combined stimulation of nociceptors and la afferents (when ankle tendons were vibrated) could result from the convergence of these afferents at the spinal level. This could certainly lead to the hypothesis that individuals suffering from lower limb pain present alterations of the postural control mechanisms; especially populations already at risk of falling (for example, frail elderly) or populations suffering from concomitant lower limb pain and sensory deficits (for example, diabetic polyneuropathy).
\end{abstract}




\section{Background}

The control of an upright standing posture is an essential motor behavior; it offers the stable platform for several goal-directed movements that we perform with the upper limbs. External and internal forces acting on the erect body create destabilizing events yielding postural oscillations [1]. The postural control system regulates these body oscillations by maintaining the vertical alignment of the body segments. The effectiveness of the postural control system depends on the availability and reliability of the visual, vestibular and somatosensory (cutaneous and proprioceptive) afferent inputs and the motor outputs [2]. When any of these components is altered, body sway generally increases and postural muscle activities increase concurrently in order to maintain postural equilibrium [3]. For example, withdrawal of visual information [4], co-vibrating the ankle tendons (falsifying or masking the muscle spindle inputs from muscles lengthening) [5-7] or providing inaccurate ankle sensory information by sway-referencing the support surface [8,9] all yield increases in body oscillations.

Large diameter myelinated afferents provide the primary source of lower limb proprioceptive information (e.g. pressure and position sense receptors) for maintaining an erect stance. Small diameter afferents also can influence motor behaviors. Noxious stimuli (chemical, thermal or mechanical) activate nociceptors which are the peripheral endings of small diameter primary sensory neurons. Sherrington [10] first proposed that the withdrawal of a limb from a noxious stimulus is controlled by the flexor reflex afferent system. The flexor reflex afferent system is characterized by descending commands from supraspinal centers and by convergence of small and large diameter afferent inputs to interneuron pathways [11,12]. The excitability of the segmental gamma and alpha motoneurone pools is influenced by the stimulation of nociceptors [13-15], highlighting the possible role of the high threshold small diameter afferents in the control of movement.

Chronic pain activity induces steady variations in interneuronal transmission that could alter motor strategies [16]. For instance, prolonged exposure to nociceptive stimulations from the skin or sore muscles affect the perception of a produced torque [17]. Weerakkody et al. [17] reported that subjects overestimated the level of torque generated by a limb affected by a pain stimulation. Also, pain can induce a distortion of the body image, leading to a biased estimation of the body position in space [18]. Despite these evidences showing the effects of painful stimulations on the sensorimotor system, their effects on the postural control system have not been well documented. A better knowledge of how nociceptive mechanisms affect the control of posture could help for developing new and adapted therapeutic approaches for patients presenting acute and chronic pain.

In the present study, two distinct experiments were performed: first, a 30-s cutaneous non-noxious heat stimulation $\left(40^{\circ} \mathrm{C}\right)$ was used to stimulate small diameter afferents yielding a gentle warmth sensation; second, a 30s cutaneous noxious heat stimulation $\left(45^{\circ} \mathrm{C}\right)$ was used to stimulate small diameter afferents yielding a pain sensation described as a distinct burning and prickling experience. These two experiments were performed to determine if the control of an upright stance can be affected by i) a gentle stimulation of the cutaneous thermal receptors and ii) a noxious heat stimulation of the skin receptors. The center of pressure displacements were analyzed to examine how both cutaneous stimulations affected the regulation of quiet standing. Subjects were tested with and without vision and when ankle proprioception (only without vision) was perturbed using the covibration technique $[6,19]$. This was done in order to reveal if warmth (Experiment 1) or painful sensations (Experiment 2) can alter the multisensory integration underlying the postural control system. It was expected that i) only the noxious stimulus would increase the postural oscillations, ii) the deterioration of the postural oscillations would be exacerbated by the withdrawal of vision due to the importance of the visual information in the control of posture and iii) the co-vibration of the ankle tendons would exacerbate the increased postural oscillations due to the convergence of the small and large diameter afferents at the spinal level [20].

\section{Methods \\ Experiment I}

Subjects and apparatus

Ten healthy male subjects participated in the study (age: $24.5 \pm 2.7$ years; height: $178 \pm 7 \mathrm{~cm}$, body weight: $81.1 \pm$ $8.5 \mathrm{Kg}$ ). The subjects were recruited at Laval University and had no evidence of gait, postural or musculo-skeletal abnormalities. Informed consent was obtained from each subject according to university protocols. Postural stability was evaluated with the help of a force platform (AMTI OR6-5-1 model). Force and moment components were amplified (Ectron 563H) prior to be sampled at $200 \mathrm{~Hz}$ (12 bit A/D conversion). Data were digitally filtered with a fourth-order Butterworth filter $(7 \mathrm{~Hz}$ low pass cut-off frequency with dual-pass to remove phase shift). The antero-posterior (A-P) and medio-lateral (M-L) coordinates of the center of foot pressure $(\mathrm{CP})$ were derived from filtered data. The tendon vibration technique was used to alter the proprioceptive information of the ankle muscles. Four vibrators were fixed with rubber bands to the subject's ankles on the tendons of both soleus and tibialis anterior muscles. When applied bilaterally to both antagonistic ankle muscles, the vibration gives instant rise to a 
background noise and deprives subjects from relevant proprioceptive messages generated by body oscillations $[19,21]$. Each vibrator $(\mathrm{n}=4)$ consisted of a plastic cylinder $(10 \mathrm{~cm}$ long, $3 \mathrm{~cm}$ diameter) containing a DC motor. The vibratory stimulation was induced by the DC motor rotating an unbalanced small mass at $80 \mathrm{~Hz}$; it produced a mechanical oscillation of $3 \mathrm{~mm}$ amplitude.

\section{Postural stability protocol}

We evaluated postural sway for six different conditions. Standing eyes open, eyes closed and eyes closed with covibration were performed with and without bilateral nonnoxious heat stimulation of the lower limbs. Subjects were instructed to stand upright as still as possible using a standardized stance on the force platform: they had their feet $10 \mathrm{~cm}$ apart, centered on the force platform. They stood barefoot on the force platform with their arms comfortably lying on each side and were instructed to fixate a point located 4 meters in front of them. Each trial lasted 30 seconds. A series of four trials was performed for each control condition (without heat stimulation): eyes open, eyes closed and eyes closed with co-vibration. The order of presentation of conditions was randomized across subjects. Three randomly presented series of four trials also were performed with the heat stimulation. Half of the subjects started with the control no-heat stimulation conditions and the other half started with the heat stimulation conditions. Rest periods of 20 seconds were provided between each trial; five-minute periods were provided between conditions. Subjects were told they could interrupt the experimental session at any time if they felt the need.

\section{Non-noxious heat stimulation protocol}

The technique for inducing the non-noxious heat stimulation was inspired from the work of Craig and colleagues $[22,23]$. Heat stimulation was induced bilaterally on the calves of the subject with two 15 by $14.5 \mathrm{~cm}$ thermal grills (Fig. 1). Each thermal grill consisted of 10 parallel copper bars $(10 \mathrm{~mm}$ diameter) spaced by $0.5 \mathrm{~cm}$. The bars were connected to each other with plastic tubing. Both thermal grills were fixed on the calves of the subjects with Velcro bands. Non-noxious heat stimuli were produced by the conduction of hot water circulating through the copper bars. Temperature of the grills was maintained at $40.0^{\circ} \mathrm{C}$ $\left( \pm 0.3^{\circ} \mathrm{C}\right)$ and induced a perception of gentle warmth. The temperature was monitored with a thermal probe sensor (Cole-Parmer thermometer, 08402-00, USA). Immediately after the fixation of the thermal grills, subjects were tested for postural stability. Subjects had the thermal grills fixated to their calves approximately $20 \mathrm{~s}$ before the beginning of a block of heat stimulation trials. Completion of a block of four postural trials (with the thermal stimulus) took less than four minutes. For control conditions (no

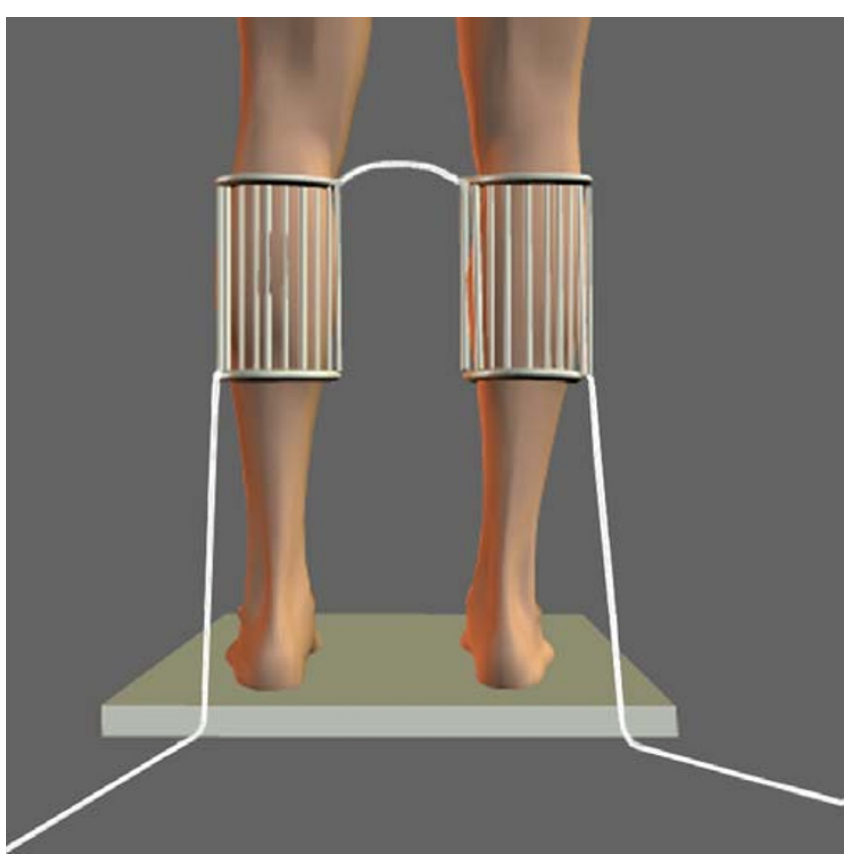

Figure I

thermal stimulus), the thermal grills were fixed on the calves without hot water.

Non nociceptive receptors, both $\mathrm{A} \delta$ and $\mathrm{C}$ warm receptors, that are responsible for the gentle sensation of warmth presumably were stimulated by the non-noxious heat stimuli $\left(40 \pm 0.3^{\circ} \mathrm{C}\right)[24]$.

\section{Pain intensity assessment}

A visual analogue scale of $10 \mathrm{~cm}$ was used to rate the sensory intensity to noxious heat stimulation. The scale had two descriptors on each end: at the left end 'no pain' and at the right end 'worst imaginable pain'. Pain was defined as any uncomfortable sensation (particularly burning and prickling) even if the stimulus was tolerable. Subjects rated their perception of pain intensity by drawing a line on the visual analogue scale at the beginning and at the end of each trial. Pain for a given trial was defined as the mean visual analogue score of the beginning and the end of that trial. Visual analogue scales have been validated for experimental pain [25].

\section{Data reduction}

Range and standard deviation of the CP trajectory, standard deviation of the $\mathrm{CP}$ velocity and mean $\mathrm{CP}$ velocity along the A-P and M-L axes were calculated. The range of the CP displacement indicates the average minimum and maximum excursion of the $\mathrm{CP}$ within the base of support. 
The calculation of the standard deviation of the signal provides a measure of amplitude variability of the $\mathrm{CP}$ around the mean position. The CP velocity was calculated by the first time derivative of the CP signal using a $200 \mathrm{~ms}$ window. The standard deviation of the $\mathrm{CP}$ velocity can be used to represent the variability of the rate of change of the $\mathrm{CP}$ required to maintain an upright stance. The mean $\mathrm{CP}$ velocity represents the total distance covered by the $\mathrm{CP}$ (total sway path) divided by the duration of the sampled period and constitutes a good index of the amount of activity required to maintain stability [26].

\section{Statistical analysis}

Dependent variables were all submitted to a 2-way ANOVA ( 2 Heat $\times 3$ Sensory conditions) with repeated measures on both factors. If a main effect of Sensory conditions or a Pain X Sensory interaction was found, a decomposition of the main effect or interaction was performed with pre-planned comparisons. The level of significance was set at $\mathrm{p}<0.05$.

\section{Experiment 2}

Subjects and apparatus

Ten healthy male subjects participated in the study (age: $25.7 \pm 2.7$ years; height: $177 \pm 8 \mathrm{~cm}$, body weight: $75.9 \pm$ $10.2 \mathrm{Kg}$ ). The subjects were recruited at Laval University and had no evidence of gait, postural or musculo-skeletal abnormalities. Informed consent was obtained from each subject according to university protocols. The apparatus was the same as in the first experiment.

\section{Postural stability protocol}

Postural sway was evaluated for six different conditions: standing eyes open, eyes closed and eyes closed with covibration were performed with and without bilateral pain stimulation of the lower limbs. Each trial lasted 30 seconds. A series of eight trials was performed for each control condition (without heat stimulation): eyes open, eyes closed and eyes closed with co-vibration. The order of presentation of conditions was randomized across subjects. Three randomly presented series of eight trials also were performed with the pain stimulation. Half of the subjects started with the control no-pain stimulation conditions and the other half started with the pain stimulation conditions. Rest periods of 20 seconds were provided between each trial; five-minute periods were provided between conditions. For the pain stimulation conditions, an additional five-minute rest period was provided after the fourth trial. During this period, the pain stimulus was removed. Hence, the painful conditions were performed in two blocks of four trials (total of eight trials).

\section{Pain stimulation protocol}

The painful stimulation was delivered with the grill described in experiment 1 . Temperature of the grills was maintained at $45.1^{\circ} \mathrm{C}\left( \pm 0.3^{\circ} \mathrm{C}\right)$ with running hot water and the temperature was evaluated with a Cole-Parmer thermometer (08402-00, USA). This thermal stimulation induced a perception of heat pain. Subjects had the thermal grills fixated to their calves approximately $20 \mathrm{~s}$ before the beginning of a block of heat stimulation trials. Completion of a block of four postural trials (with the thermal stimulus) took less than four minutes. For the control conditions (no thermal stimulus), the thermal grills were fixed on the calves without hot water.

Two main groups of receptors, namely the A $\delta$ mechanothermal receptors and the $\mathrm{C}$ mechano-heat nociceptors presumably were stimulated by the pain stimulus delivered in the present experiment $\left(45.1 \pm 0.3^{\circ} \mathrm{C}\right)$. The $\mathrm{A} \delta$ mechano-thermal receptors respond to noxious heating and are characterized by a threshold ranging from 37 to $47^{\circ} \mathrm{C}$ [27]. The $\mathrm{C}$ mechano-heat nociceptors have thermal threshold ranging from 38 to $49^{\circ} \mathrm{C}$ with mean values around $44^{\circ} \mathrm{C}$ [28].

\section{Pain intensity assessment}

As for experiment 1, a VAS was used to evaluate the perception of pain induced by the thermal stimulus.

\section{Data reduction}

Range and standard deviation of the CP trajectory, standard deviation of the $\mathrm{CP}$ velocity and mean $\mathrm{CP}$ velocity along the A-P and M-L axes were calculated similarly to experiment 1 .

\section{Statistical analysis}

Dependent variables were all submitted to a 2-way ANOVA (2 Pain $\times 3$ Sensory conditions) with repeated measures on both factors. If a main effect of Sensory conditions or a Pain X Sensory interaction was found, a decomposition of the main effect or interaction was performed with pre-planned comparisons. The level of significance was set at $\mathrm{p}<0.05$.

\section{Results}

\section{Experiment I: The effects of the non-noxious heat} stimulation on the control of posture

Perceived pain intensity

For the non-noxious heat stimulation, no subject reported any pain $(\mathrm{VAS}=0)$. All subjects described the non-noxious heat stimulation as a gentle sensation of warmth.

\section{Postural analyses}

Table 1 presents the mean and standard deviation for all computed variables for the control and non-noxious heat stimulation conditions. The F-values for the ANOVAs also are presented. For all dependent variables, the ANOVAs revealed main effects of the Sensory conditions (ps < 0.01 ) but no main effect of the non-noxious Heat stimu- 
Table I: Mean (standard deviation) and ANOVA results of the center of foot pressure parameters for the various sensory conditions with and without a non-noxious heat stimulation.

\begin{tabular}{|c|c|c|c|c|c|c|c|c|c|}
\hline \multirow[t]{2}{*}{ Parameters } & \multicolumn{2}{|c|}{ Vision } & \multicolumn{2}{|c|}{ No Vision } & \multicolumn{2}{|c|}{ No Vision co-Vibration } & \multicolumn{3}{|c|}{ F-Values } \\
\hline & No Heat & Heat & No Heat & Heat & No Heat & Heat & Heat & Sensory & Interaction \\
\hline Range A-P (mm) & $14.5(4.5)$ & $14.4(3.6)$ & $18.5(6.3)$ & $18.4(5.1)$ & $27.6(10.9)$ & $27.3(7.7)$ & 0.04 & $18.69 \neq$ & 0.00 \\
\hline Range M-L (mm) & $7.2(3.2)$ & $8.2(4.0)$ & $9.2(4.7)$ & $9.1(4.9)$ & $13.2(6.2)$ & $13.2(5.2)$ & 0.79 & $21.17 \ddagger$ & 0.89 \\
\hline Standard Deviation A-P (mm) & $3.3(1.2)$ & $3.1(0.9)$ & $3.6(1.2)$ & $3.6(1.1)$ & $4.8(1.7)$ & $5.0(1.4)$ & 0.03 & $10.30 \dagger$ & 1.23 \\
\hline Standard Deviation M-L (mm) & $1.5(0.7)$ & $1.7(1.0)$ & $1.8(0.9)$ & $1.7(1.0)$ & $2.3(1.0)$ & $2.4(1.0)$ & 1.19 & $14.63 \ddagger$ & 0.83 \\
\hline Standard Deviation of the CP Velocity A-P $(\mathrm{mm} / \mathrm{s})$ & $5.8(1.1)$ & $6.7(1.8)$ & $9.5(2.7)$ & $10.0(2.1)$ & $16.6(6.3)$ & $16.7(5.8)$ & 2.11 & $26.77 \ddagger$ & 0.27 \\
\hline Standard Deviation of the CP Velocity M-L $(\mathrm{mm} / \mathrm{s})$ & $2.9(1.0)$ & $3.4(1.2)$ & $4.3(2.2)$ & $4.1(1.7)$ & $8.4(4.7)$ & $8.6(4.4)$ & 1.50 & $20.35 \ddagger$ & 1.36 \\
\hline Mean Velocity A-P (mm/s) & $4.7(0.9)$ & $5.5(1.5)$ & $7.5(2.0)$ & $8.1(1.6)$ & $13.1(4.8)$ & $13.3(4.3)$ & 4.35 & $28.60 \ddagger$ & 0.18 \\
\hline Mean Velocity M-L (mm/s) & $2.4(0.8)$ & $2.7(0.9)$ & $3.3(1.6)$ & $3.2(1.2)$ & $6.6(3.6)$ & $6.8(3.4)$ & 1.74 & $20.31 \ddagger$ & 1.19 \\
\hline
\end{tabular}

Note: $*, \dagger$ and $\ddagger$ indicate a significant difference at $p<0.05, p<0.01$ and $p<0.001$, respectively.

Table 2: Mean (standard deviation) and ANOVA results of the center of foot pressure parameters for the various sensory conditions with and without cutaneous pain.

\begin{tabular}{|c|c|c|c|c|c|c|c|c|c|}
\hline \multirow[t]{2}{*}{ Parameters } & \multicolumn{2}{|c|}{ Vision } & \multicolumn{2}{|c|}{ No Vision } & \multicolumn{2}{|c|}{ No Vision co-Vibration } & \multicolumn{3}{|c|}{ F-Values } \\
\hline & No Pain & Pain & No Pain & Pain & No Pain & Pain & Pain & Sensory & Interaction \\
\hline Range A-P (mm) & $16.4(5.5)$ & $19.8(6.0)$ & $19.7(4.6)$ & $24.9(7.3)$ & $30.6(11.5)$ & $37.7(12.3)$ & $13.96 \dagger$ & $33.64 \neq$ & 2.08 \\
\hline Range M-L (mm) & II.3 (3.9) & $13.4(5.4)$ & $12.7(5.6)$ & $16.3(7.3)$ & $21.4(10.3)$ & $21.8(10.1)$ & $16.50 \dagger$ & $18.95 \neq$ & 2.19 \\
\hline Standard Deviation A-P (mm) & $3.5(1.3)$ & $4.0(1.1)$ & $3.9(1.1)$ & $4.6(1.4)$ & $5.8(2.3)$ & $6.7(2.4)$ & $7.01 *$ & $19.62 \ddagger$ & 0.66 \\
\hline Standard Deviation M-L (mm) & $2.2(0.8)$ & $2.6(1.0)$ & $2.5(1.1)$ & $3.1(1.3)$ & $3.9(1.8)$ & $3.9(1.8)$ & $15.23 \dagger$ & $18.33 \neq$ & 2.66 \\
\hline Standard deviation of the CP Velocity A-P $(\mathrm{mm} / \mathrm{s})$ & $7.1(1.2)$ & $8.6(2.9)$ & $10.2(2.1)$ & $12.0(3.2)$ & $19.6(6.3)$ & $25.4(7.1)$ & $37.42 \ddagger$ & $59.40 \neq$ & $5.87 *$ \\
\hline Standard deviation of the CP Velocity M-L $(\mathrm{mm} / \mathrm{s})$ & $5.1(1.6)$ & $6.3(2.6)$ & $5.9(2.9)$ & $7.4(3.5)$ & $13.3(7.1)$ & $13.9(6.8)$ & $9.70 *$ & $23.40 \neq$ & 0.67 \\
\hline Mean Velocity A-P $(\mathrm{mm} / \mathrm{s})$ & $5.7(1.0)$ & $6.9(2.0)$ & $8.1(1.7)$ & $9.5(2.6)$ & $15.5(5.0)$ & $20.0(5.5)$ & $58.84 \ddagger$ & $56.34 \ddagger$ & $5.61 *$ \\
\hline Mean Velocity M-L (mm/s) & $4.0(1.2)$ & $4.9(1.9)$ & $4.6(2.3)$ & $5.8(2.6)$ & $10.4(5.5)$ & $11.0(5.3)$ & $10.10 *$ & $23.21 \neq$ & 0.43 \\
\hline
\end{tabular}

Note: $*$, and $\ddagger$ indicate a significant difference at $p<0.05, p<0.01$ and $p<0.001$, respectively.

lation and no interaction (ps > 0.05). Withdrawal of vision increased the range and mean velocity of the $\mathrm{CP}$ oscillations and increased the standard deviation of the $\mathrm{CP}$ velocity along both axes (ps $<0.05)$. Co-vibrating the ankle tendon without vision yielded greater standard deviation and range of the $\mathrm{CP}$ oscillations and greater standard deviation of the CP velocity and mean CP velocity along both axes compared to the no vision condition (ps<0.05).

\section{Experiment 2: The effects of the pain stimulation on the control of posture}

Perceived pain intensity

All subjects reported no pain for the control conditions. For the pain conditions, the mean visual analogue score was $46 \mathrm{~mm}(\mathrm{SD}=17), 46(\mathrm{SD}=20)$ and $45(\mathrm{SD}=19)$ for the vision, no vision and no vision co-vibration conditions, respectively. The visual analogue scores of heat pain were not different for all experimental pain conditions ( $\mathrm{p}$ $>0.05$ ). All subjects described the noxious heat stimuli as a distinct burning, prickling sensation.

\section{Postural analyses}

Table 2 presents the mean and standard deviation for all computed variables. The F-values for the ANOVAs also are presented.

A preliminary analysis of the data was performed using simple linear regression with trial number as the independent variable and each CP parameter as the dependent variable. For all dependent variables, there was no sign of habituation: each adjusted $\mathrm{R}^{2}$ value was lower than 0.02 . To illustrate this absence of habituation, Fig. 2 presents CP oscillations, for one subject, for all trials without vision with and without a noxious heat stimulation. Clearly, the effect of the noxious stimulation is observed across all trials and there is no trend towards an increased or a decreased of the range of the CP oscillations when the pain stimulation is applied.

The range of the $\mathrm{CP}$ oscillations increased for the Pain conditions for both the A-P and M-L axes ( $\mathrm{ps}<0.01$ ). The ANOVAs also showed a main effect of Sensory conditions (ps $<0.001$ ) : for both axes, the range of the CP was greater for the no vision compared to the vision condition and also greater for the no vision co-vibration compared to the 
no vision condition ( $\mathrm{ps}<0.05$ ). No interaction of Pain by Sensory conditions was observed (ps > 0.05).

For the standard deviation of the $\mathrm{CP}$, the ANOVAs showed an increased CP standard deviation for the Pain conditions for both axes (ps < 0.05). For both axes, the ANOVAs showed a main effect of Sensory conditions (ps < 0.001). The standard deviation of the $\mathrm{CP}$ was greater for the no vision compared to the vision condition and also greater for the no vision co-vibration compared to the no vision condition ( $\mathrm{ps}<0.05)$. No interaction of Pain by Sensory conditions was observed ( $\mathrm{ps}>0.05$ ).

For the standard deviation of the $\mathrm{CP}$ velocity along the A$\mathrm{P}$ axis, the ANOVA revealed an interaction of Pain by Sensory conditions $(\mathrm{p}<0.05)$ and main effects of Pain $(\mathrm{p}<$ $0.05)$ and Sensory conditions $(\mathrm{p}<0.001)$. Decomposition of the interaction showed that the increase of the standard deviation of the CP velocity from vision to no vision was similar with and without pain $(40 \%$ and $44 \%$ with and without pain, respectively; planned comparison, $p>0.05)$. The increase of the standard deviation of the CP velocity from no vision to no vision co-vibration, however, was greater for the Pain conditions than for the control no-pain conditions $(112 \%$ and $92 \%$ with and without pain, respectively; planned comparison, p < $0.05)$. The ANOVA revealed that the standard deviation of the $\mathrm{CP}$ velocity along the $\mathrm{M}-\mathrm{L}$ axis increased for the Pain conditions $(\mathrm{p}<0.05)$. The main effect of Sensory conditions also was statistically significant $(p<0.001)$, but the interaction of Pain $x$ Sensory conditions was not ( $p>0.05$ ). The standard deviation of the CP velocity along the $\mathrm{M}$-L axis increased for the no vision co-vibration condition compared to the vision and no vision conditions ( $\mathrm{ps}<0.05)$.

Fig. 3 presents the mean $\mathrm{CP}$ velocity along the A-P and M$\mathrm{L}$ axes. For the A-P axis, the ANOVA revealed an interaction of Pain by Sensory conditions $(p<0.05)$ and main effects of Pain $(\mathrm{p}<0.001)$ and Sensory conditions $(p<0.001)$. A decomposition of the interaction showed that the increase of the CP mean velocity from vision to no vision was similar with and without pain (37\% and $43 \%$ with and without pain, respectively; planned comparison, $\mathrm{p}>0.05$ ). The increase of the $\mathrm{CP}$ mean velocity from no vision to no vision co-vibration, however, was greater for the Pain conditions than for the control nopain conditions $(110 \%$ and $90 \%$ with and without pain, respectively; planned comparison, $\mathrm{p}<0.05$ ). For the $\mathrm{M}-\mathrm{L}$ axis, the ANOVA showed an increased CP mean velocity for the Pain conditions $(\mathrm{p}<0.05)$ and a main effect of Sensory conditions $(\mathrm{p}<0.001)$, but no interaction $(\mathrm{p}>$ 0.05). The mean CP velocity along the M-L axis increased for the no vision co-vibration condition compared to the vision and no vision conditions ( $\mathrm{ps}<0.05$ ).

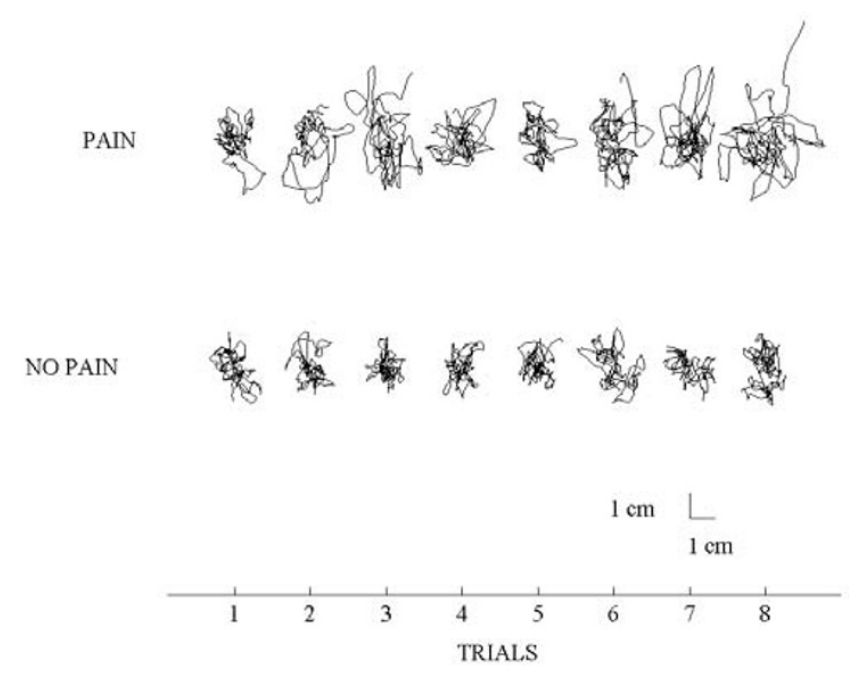

Figure 2

Overall, the noxious heat stimulation of the lower limbs yielded increased range and standard deviation of the $\mathrm{CP}$ trajectory, standard deviation of the CP velocity and mean velocity of the postural oscillations along both axes.

\section{Discussion}

The present study examined the postural stability of young adults in the presence of non-noxious and noxious heat stimulations under various sensory conditions. Both with and without the heat stimulations, the $\mathrm{CP}$ data observed for the different sensory conditions were consistent with previous findings $[4,6,7]$ More specifically, withdrawal of visual information and co-vibrating ankle tendons both yielded increases in body oscillations.

\section{The effects of the non-noxious heat stimulation on the control of posture}

Results of experiment 1 showed that the non-noxious heat stimulation (warm receptors) did not yield a pain sensation and did not affect the control of posture. These results are in agreement with recent neurophysiological evidences showing that the stimulation of warm receptors has only mild effects on spinal interneuron excitability. For example, Plaghki et al[24] have reported that, compared to noxious stimuli, non-noxious radiant heat stimuli yielded a moderate facilitation of the withdrawal flexor reflex (15-20\% compared to $150-200 \%$ for the noxious stimuli). Moreover, in a literature review, Schomburg [20] has reported that thermal stimulation below the nocicep- 

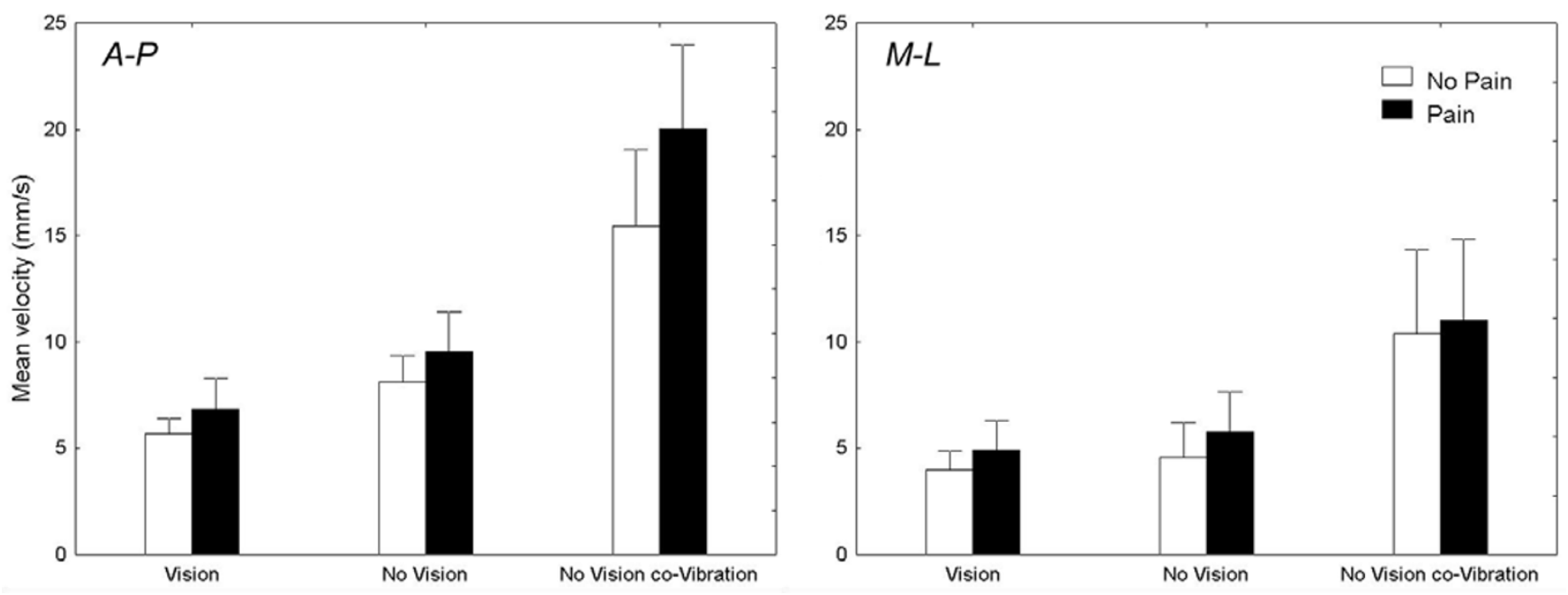

Figure 3

tive threshold failed to elicit synaptic effects in $\alpha$-motoneurons or facilitatory effects onto reflex pathways from group Ib or II muscle afferents or mechanoreceptive cutaneous afferents. In the present experiment, the small effects presumably induced by the non-noxious heat stimulation at the segmental level were not sufficient to influence the maintenance and control of the upright stance.

\section{The effects of the pain stimulation on the control of posture}

The aim of experiment 2 was to document how a noxious heat stimulation inducing a perception of pain would alter the regulation of postural stability in normal quiet standing. In the present experiment, despite the presence of a pain sensation, subjects maintained an erect posture without the presence of any withdrawal movements. The overall effect of the noxious heat stimulation clearly stresses its negative impact on the postural control system. It appears that the action/detection properties of the postural control system necessary to regulate quiet standing were affected by the cutaneous noxious heat stimulation (thermal nociceptors). The present results are at odd with recent findings showing that the regulation of the upright stance was not affected by pain reduction in patients with knee osteoarthritis[29]. Hassan et al. [29], however, have argued that their measure of postural stability might have been insensitive to pain reduction. The effect of pain reduction also could have been attenuated by the decrease of the knee proprioception induced by injection of the anaesthetic substance. In the present experiment, young healthy individuals were tested for postural stability with and without noxious heat stimulation inducing a perception of pain. Thus, any confounding effect of pain with a subjacent pathology is a priori excluded.

Results observed for the CP fluctuations along the M-L direction suggest that pain stimulation clearly modified the upright standing behaviors. The increased variability of the CP amplitude (around the mean position of the CP during a trial) and CP velocity (around the mean velocity of the CP during a trial), the increased range of the postural oscillations and the increased CP mean velocity all suggest a less stable and more variable behavior with than without a noxious heat stimulation. For the A-P direction, subjects also exhibited more and faster postural oscillations, greater range and variability of the CP amplitude and velocity with than without a noxious heat stimulation of the lower limbs. These effects could be associated with the control of the postural oscillations required to compensate the motor and/or sensory changes induced by the noxious heat stimulation possibly through the activation of $\mathrm{A} \delta$ mechano-thermal and $\mathrm{C}$ mechano-heat receptors (nociceptive thermoreceptors).

There are several possible interpretations to explain the $\mathrm{CP}$ changes observed with the pain stimulation. One possibility resides in the changes occurring at the spinal level induced by nociceptive afferents (e.g. reflex inhibition of the motoneuron pools, presynaptic excitability changes of Ia fibers or increased sensitivity of the muscle spindles) $[13,14,16,30]$. A second possibility could be related to changes in the excitability of the motor cortex [31] or in the sensory integration processes [18]. The increased loss of stability associated with the noxious heat 
stimulation could have been the result of an altered central motor command to the periphery or a reduced accuracy in the integration of the sensory information which ultimately led to a biased estimation of the whole body position in space. For balance control, the precision of the compensatory ankle torques to maintain the vertical alignment of the body largely depends upon the incoming sensory information. Both of these possibilities could alter force production or sensory detection capabilities yielding greater and faster displacements of the CP. The present results, however, do not allow to extrapolate on the exact locus of the observed effects of the noxious pain stimulation.

Vibration stimuli can reduce the effects of a painful stimulation [32]. This presumably occurs through inhibition of small diameter afferents in the dorsal horn of the spinal cord by large myelinated fibers [33]. In the present experiment, the tendon co-vibration did not modify the perception of the painful stimulation. This absence of pain relief was certainly related to the short duration of the applied co-vibration [34]. Also, it could be that the frequency of the vibration was below that necessary to alleviate pain [35]. The ankle tendon co-vibration technique accentuated the deteriorating effects of the painful stimulation on the mean velocity of the $\mathrm{CP}$ and the standard deviation of the $\mathrm{CP}$ velocity (A-P axis). These variables describe important features of the CP oscillations. First, the $\mathrm{CP}$ mean velocity supposedly reflects the amount of regulatory balancing activity that is needed to maintain an upright posture [26] and can be used to discriminate the postural behaviors between symptomatic population [36]. Second, the standard deviation of the CP velocity can be used as an indicator of the variability in the rate of change of $\mathrm{CP}$ and has showed good intrasubject consistency as well as a high sensitivity to visual deprivation [37]. For the M-L axis, pain yielded an increased range, standard deviation, mean velocity of the $\mathrm{CP}$ oscillations and standard deviation of the $\mathrm{CP}$ velocity but this effect was not different across all sensory conditions. The vibrated muscles (tibialis anterior and triceps surae) act mainly to regulate postural oscillations along the A-P axis. This suggests that the negative effects of pain receptors on the postural control system are exacerbated when proprioceptive information from the relevant muscle to the control of posture is masked or falsified. Schomburg [20] has argued that the convergence of small and large diameter afferents (stimulated respectively by the pain stimulation and the tendon co-vibration technique in our experiment) occurs at the spinal level of the sensorimotor system. This convergence of polymodal sensory information could produce the observed deteriorating effects of the combined nociceptive and Ia afferents on the motor system. An increase in the amplitude of the nociceptive leg flexion reflex by the application of tendon vibration has been observed previously by other authors [38].

Overall, the effects of the painful stimulation were not exacerbated when vision was withdrawn. This suggests that the visual information could not compensate for the deteriorating effects of the pain stimulation on the postural control system. It is possible, however, that the contribution of vision to reduce painful stimulation would be different at higher perceived pain intensities. Also, our pain stimulation was continuous throughout the 30-s trial; the effect of a short duration painful event on postural control remains to be determined.

\section{Conclusion}

The results presented in this study show that a painful sensation alters the control of an upright stance. Furthermore, a non-noxious heat stimulation of the small diameter afferents is not sufficient to influence the control of posture. To our knowledge no association has been made between pain sensation and the deterioration of the postural control mechanisms. This could certainly lead to the hypothesis that individuals suffering from lower limb pain present alterations of the postural control mechanisms; especially populations already at risk of falling (for example, frail elderly) or populations suffering from concomitant lower limb pain and sensory deficits (for example, diabetic polyneuropathy).

\section{Competing interests}

None declared.

\section{Author's contributions}

JSB and PC participated in all aspects of the study. NT participated in the design and coordination of the study, interpreted the results and drafted the manuscript. All authors read and approved the final manuscript.

\section{Acknowledgments}

This study was supported in part by the NSERC-Canada, Égide-France and ClHR-FCQ funds. Special thanks to Drs Nicole Paquet, Laurence Mouchnino and Douglas E Young for several insightful comments on a previous version of this manuscript. Special thanks also to Gilles Bouchard and Marcel Kaszap for technical and programming expertise and to Guillaume Desroches for his help in collecting the data. This experiment is part of a research program that has been approved by the local ethics committee.

\section{References}

I. Hunter IW and Kearney RE: Respiratory components of human postural sway. Neurosci Lett I98I, 25: I55-I59.

2. Massion J: Postural control system. Curr Opin Neurobiol 1994, 4:877-887.

3. Dietz V: Human neuronal control of automatic functional movements: Interaction between central programs and afferent input. Physiol. Rev. 1992, 72:33-69.

4. Brandt T, Paulus $W$ and Straube A: Vision and posture. Disorders of Posture and Gait Edited by: Bles W and Brandt T. Amsterdam, Elsevier Science Publishers BV (Biomedical Division); 1986:157-175. 
5. Eklund G: General features of vibration-induced effects on balance. Ups J Med Sci 1972, 77: I I2-I 24.

6. Hayashi R, Miyake $A$ and Watanabe $S$ : The functional role of sensory inputs from the foot: stabilizing human standing posture during voluntary and vibration-induced body sway. Neurosci Res 1988, 5:203-213

7. Hay L, Bard C, Fleury M and Teasdale N: Availability of visual and proprioceptive afferent messages and postural control in elderly adults. Exp Brain Res 1996, I08:129-139.

8. Manchester D, Woollacott $\mathrm{M}$, Zederbauer-Hylton $\mathrm{N}$ and Marin $\mathrm{O}$ : Visual, vestibular and somatosensory contributions to balance control in the older adult. J. Gerontol.: Med. Sc. 1989, 44:MII8-127.

9. Horak FB and Diener HC: Cerebellar control of postural scaling and central set in stance. J. Neurophysiol. 1994, 72:470-493.

10. Sherrington CS: Flexion-reflex of the limb, crossed extension reflex, and reflex stepping and standing. J Physiol 1910, 40:28- $|2|$.

II. Lundberg A: Multisensory control of spinal reflex pathways. Prog Brain Res 1979, 50: I -28.

12. Lundberg $A$, Malmgren $K$ and Schomburg ED: Reflex pathways from group II muscle afferents. 3. Secondary spindle afferents and the FRA: a new hypothesis. Exp Brain Res 1987 65:294-306.

13. Matre DA, Sinkjaer T, Svensson $P$ and Arendt-Nielsen L: Experimental muscle pain increases the human stretch reflex. Pain 1998, 75:331-339.

14. Capra NF and Ro JY: Experimental muscle pain produces central modulation of proprioceptive signals arising from jaw muscle spindles. Pain 2000, 86:15I-162.

15. Rossi A, Decchi B, Dami S, Della Volpe R and Groccia V: On the effect of chemically activated fine muscle afferents on interneurones mediating group I non-reciprocal inhibition of extensor ankle and knee muscles in humans. Brain Res 1999 8I5: I06-II0.

16. Rossi $A$ and Decchi $B$ : Changes in lb heteronymous inhibition to soleus motoneurones during cutaneous and muscle nociceptive stimulation in humans. Brain Res 1997, 774:55-61.

17. Weerakkody N, Percival P, Morgan DL, Gregory JE and Proske U: Matching different levels of isometric torque in elbow flexor muscles after eccentric exercise. Exp Brain Res 2003, 149: I4I-I 50.

18. Gandevia SC and Phegan CM: Perceptual distortions of the human body image produced by local anaesthesia, pain and cutaneous stimulation. J Physiol 1999, 5 I 4 ( Pt 2):609-616.

19. Pyykko I, Jantti $P$ and Aalto $H$ : Postural control in elderly subjects. Age Ageing 1990, 19:2 15-221.

20. Schomburg ED: Spinal sensorimotor systems and their supraspinal control. Neurosci Res 1990, 7:265-340.

21. Teasdale $N$ and Simoneau M: Attentional demands for postural control: the effects of aging and sensory reintegration. Gait Posture 2001, 14:203-210.

22. Craig $A D$ and Bushnell $M C$ : The thermal grill illusion: unmasking the burn of cold pain. Science 1994, 265:252-255.

23. Craig AD, Reiman EM, Evans $A$ and Bushnell MC: Functional imaging of an illusion of pain. Nature 1996, 384:258-260.

24. Plaghki L, Bragard D, Le Bars D, Willer JC and Godfraind JM: Facilitation of a nociceptive flexion reflex in man by nonnoxious radiant heat produced by a laser. I Neurophysiol 1998, 79:2557-2567.

25. Price DD, McGrath PA, Rafii A and Buckingham B: The validation of visual analogue scales as ratio scale measures for chronic and experimental pain. Pain 1983, 17:45-56.

26. Maki BE, Holliday PJ and Fernie GR: Aging and postural control. A comparison of spontaneous- and induced-sway balance tests. J Am Geriatr Soc 1990, 38: I-9.

27. Dubner R, Price DD, Beitel RE and Hu JW: Peripheral neural correlates of behavior in monkey and human related to sensorydiscriminative aspects of pain. Pain in the trigeminal region Edited by: Anderson D J and Matthews B. Amsterdam, Elsevier; 1977:57-66.

28. Beitel RE and Dubner R: Response of unmyelinated (C) polymodal nociceptors to thermal stimuli applied to monkey's face. J Neurophysiol 1976, 39: I I60-I I75.

29. Hassan BS, Doherty SA, Mockett S and Doherty M: Effect of pain reduction on postural sway, proprioception, and quadriceps strength in subjects with knee osteoarthritis. Ann Rheum Dis 2002, $61: 422-428$.

30. Rossi A, Decchi B and Ginanneschi F: Presynaptic excitability changes of group la fibres to muscle nociceptive stimulation in humans. Brain Res 1999, 81 8: 12-22.

31. Le Pera D, Graven-Nielsen T, Valeriani M, Oliviero A, Di Lazzaro V, Tonali PA and Arendt-Nielsen L: Inhibition of motor system excitability at cortical and spinal level by tonic muscle pain. Clin Neurophysiol 200I, I I 2:1633-164I.

32. Kakigi $\mathrm{R}$ and Shibasaki $\mathrm{H}$ : Mechanisms of pain relief by vibration and movement. J Neurol Neurosurg Psychiatry 1992, 55:282-286.

33. Melzack R and Wall PD: Pain mechanisms: a new theory. Science 1965, I50:97| I-979.

34. Lundeberg T, Nordemar R and Ottoson D: Pain alleviation by vibratory stimulation. Pain 1984, 20:25-44.

35. Pantaleo T, Duranti R and Bellini F: Effects of vibratory stimulation on muscular pain threshold and blink response in human subjects. Pain 1986, 24:239-250.

36. Baratto L, Morasso PG, Re C and Spada G: A new look at posturographic analysis in the clinical context: sway-density versus other parameterization techniques. Motor Control 2002, 6:246-270.

37. Geurts AC, Nienhuis B and Mulder TW: Intrasubject variability of selected force-platform parameters in the quantification of postural control. Arch Phys Med Rehabil 1993, 74: I I 44-I I 50.

38. Guieu R and Serratrice G: Identifying the afferents involved in movement-induced pain alleviation in man. Brain 1992, II 5 ( Pt 4): $1073-1079$.

\section{Pre-publication history}

The pre-publication history for this paper can be accessed here:

http://www.biomedcentral.com/1471-2474/4/23/prepub
Publish with Bio Med Central and every scientist can read your work free of charge

"BioMed Central will be the most significant development for disseminating the results of biomedical research in our lifetime. "

Sir Paul Nurse, Cancer Research UK

Your research papers will be:

- available free of charge to the entire biomedical community

- peer reviewed and published immediately upon acceptance

- cited in PubMed and archived on PubMed Central

- yours - you keep the copyright
BioMedcentral 